\title{
Explaining Left Lateralization for Words in the Ventral Occipitotemporal Cortex
}

\author{
Mohamed L. Seghier and Cathy J. Price \\ Wellcome Trust Centre for Neuroimaging, Institute of Neurology, University College London, London WC1N 3BG, United Kingdom
}

Reading is a uniquely human task and therefore any sign of neuronal activation that is specific to reading is of considerable interest. One intriguing observation is that ventral occipitotemporal (vOT) activation is more strongly left lateralized for written words than other visual stimuli. This has contributed to claims that left vOT plays a special role in reading. Here, we investigated whether left lateralized vOT responses for words were the consequence of visual feature processing, visual word form selectivity, or higher level language processing. Using fMRI in 82 skilled readers, our paradigm compared activation and lateralization for words and nonlinguistic stimuli during different tasks. We found that increased left lateralization for words relative to pictures was the consequence of reduced activation in right vOT rather than increased activation in left vOT. We also found that the determinants of lateralization varied with the subregion of vOT tested. In posterior vOT, lateralization depended on the spatial frequency of the visual inputs. In anterior vOT, lateralization depended on the semantic demands of the task. In middle vOT, lateralization depended on a combination of visual expertise in the right hemisphere and semantics in the left hemisphere. These results have implications for interpreting left lateralized vOT activation during reading. Specifically, left lateralized activation in vOT does not necessarily indicate an increase in left vOT processing but is instead a consequence of decreased right vOT function. Moreover, the determinants of lateralization include both visual and semantic factors depending on the subregion tested.

\section{Introduction}

Reading typically results in left lateralized ventral occipitotemporal (vOT) responses (Tarkiainen et al., 1999; Rossion et al., 2003; Nakamura et al., 2005; Vigneau et al., 2005). This left lateralized signature for written words has previously been explained in terms of (1) the visual attributes of the stimuli (Rossion et al., 2003), for example, increased left hemisphere activation because written words have high spatial frequencies (Hsiao and Cottrell, 2009; Woodhead et al., 2011); (2) selectivity to orthographic features and visual word forms in left vOT (Cohen et al., 2003) that may emerge during reading acquisition (Baker et al., 2007; Maurer et al., 2008); or (3) higher level language processing (Xue et al., 2005; Hunter et al., 2007; Cai et al., 2010) at the level of phonology (Maurer and McCandliss, 2007) or lexicosemantics (Sabsevitz et al., 2005; Vigneau et al., 2005). Given these different lines of evidence, we hypothesized that lateralization in vOT may depend on the combined influences of multiple factors. A second but related hypothesis is that the proposed determinants of later-

\footnotetext{
Received May 4, 2011; revised Aug. 22, 2011; accepted Aug. 25, 2011.

Author contributions: C.J.P. designed research; M.L.S. and C.J.P. performed research; M.L.S. analyzed data; M.L.S. and C.J.P. wrote the paper.

This work was supported by the Wellcome Trust and the James S. MacDonnell Foundation (conducted as part of the Brain Network Recovery Group initiative). We thank our three radiographers (Amanda Brennan, Janice Glensman, and David Bradbury); as well as (lare Shakeshaft, Laura Stewart, and Tom Schofield for their help with fMRI data collection; Caroline Ellis, Goulven Josse, and Ferath Kherif for their help with data analysis; and Hwee Ling Lee and Sue Ramsden for their valuable help setting up the fMRI database.

Correspondence should be addressed to Dr. Mohamed L. Seghier, Wellcome Trust Centre for Neuroimaging, Institute of Neurology, 12 Queen Square, London WC1N 3BG, UK. E-mail: m.seghier@fil.ion.ucl.ac.uk.

DOI:10.1523/JNEUROSCI.2238-11.2011

Copyright $\odot 2011$ the authors $\quad 0270-6474 / 11 / 3114745-09 \$ 15.00 / 0$
}

alization have varied across studies (see Table 1) because different subregions of vOT are influenced by different factors.

Our aim was to formalize and explicitly test these hypotheses in a single experiment that investigated the contribution of the following factors. First, we considered whether increased left lateralization in $\mathrm{VOT}$ for words relative to other nonlinguistic stimuli was best explained by increased left hemisphere activation, decreased right hemisphere activation, or both (Vigneau et al., 2005; Xue and Poldrack, 2007). This is important because laterality is not absolute but relative (Whitaker and Ojemann, 1977). It is therefore possible that increased left lateralization for words relative to pictures could be a consequence of more right hemisphere activation for pictures (Seghier et al., 2011a). In vOT, this could arise if recognized words put less demands on visual processing in right vOT.

Second, we investigated the visual and nonvisual factors that determined vOT lateralization by comparing left and right vOT activation in eight different conditions that systematically compared the response to stimuli with high versus low spatial frequency, which were either familiar and meaningful (words and pictures of objects) or unfamiliar and meaningless (Greek letters and pictures of nonobjects) presented under different task contexts (speech production, perceptual and semantic decisions; Fig. 1). We ensured high spatial definition for mapping laterality in different $\mathrm{vOT}$ subdivisions by generating laterality maps at the voxel level (Liégeois et al., 2002; Xue et al., 2005).

Prior studies have shown that vOT activation is left lateralized for line drawings of objects (Nakamura et al., 2005) but right lateralized for pictures of faces, with left hemisphere activation increasing with face familiarity (Laeng and Rouw, 2001; Rossion 


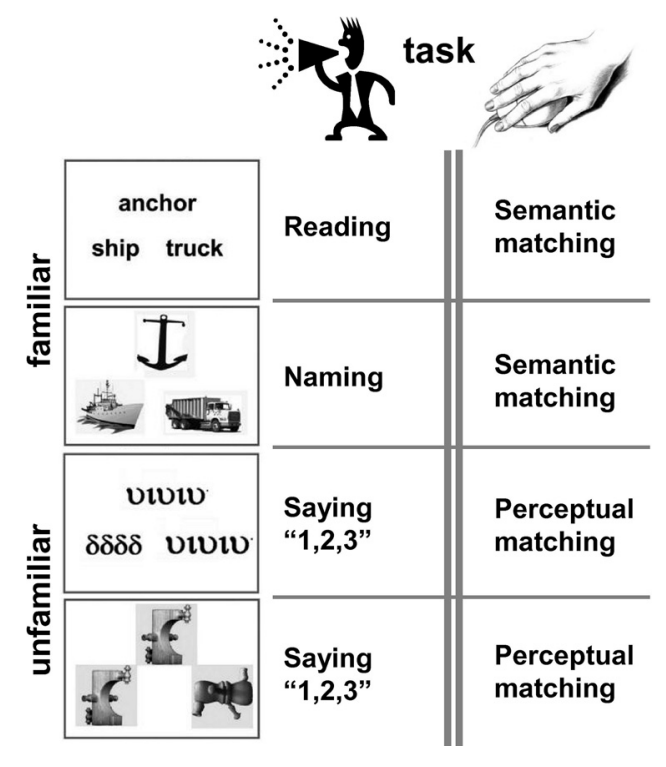

Figure 1. Illustration of tasks and stimuli used in this study (a total of eight conditions). Stimuli were presented as triads. Note that during reading and naming, there was no semantic relationship between the three stimuli.

et al., 2003; Taylor et al., 2009) and right hemisphere activation increasing with visual expertise for specific categories of objects (Gauthier et al., 1999, 2000; Tanaka and Curran, 2001). Our expectation was that the degree to which vOT activation was left lateralized for words would vary with the task and stimuli, and the specifics of these effects would differ in posterior and anterior vOT subdivisions (for detailed predictions, see Materials and Methods, below).

\section{Materials and Methods}

Subjects. Eighty-two healthy subjects (43 females, 39 males, $30.3 \pm 15$ years old, 44 right-handed, 38 left-handed or ambidextrous) gave written informed consent to participate in this study. Subjects were native English speakers with normal or corrected-to-normal vision, and had no history of difficulty learning to read or neurological or psychiatric disorders. The study was approved by the National Hospital for Neurology and Institute of Neurology Joint Ethic's Committee.

Paradigm design. There were four separate scanning runs or sessions. In two sessions, the participants made semantic and perceptual decisions, interleaved with blocks of fixation. In the other two sessions, the participants performed the four speech production tasks interleaved with blocks of fixation. The order of conditions was counterbalanced within and across session. Each session consisted of 24 blocks of stimuli of the same type/condition with an additional 12 blocks of fixation that were presented every two stimulus blocks. Each stimulus block lasted $18 \mathrm{~s}$ and consisted of four trials during which three stimuli were simultaneously presented on the screen for $4.32 \mathrm{~s}$, followed by $180 \mathrm{~ms}$ of fixation. Every two stimulus blocks, fixation continued for $14.4 \mathrm{~s}$.

Stimuli and tasks. All stimuli were presented in triads with one item (picture or letter string) above and two items below in the same format as the item above (Fig. 1). There were four different stimuli: written names of objects, photographs of objects, unfamiliar Greek strings, and unfamiliar nonobjects. We ensured that all meaningful stimuli were highly familiar perceptually (by presenting words in the widely used black font Arial and objects as grayscale photographs instead of line drawings) and conceptually (by using pictures and the written names of common objects with familiar names). To take into account any potential effect of spatial frequency on lateralized brain activity for words and pictures (Mercure et al., 2008), we also computed a mean/center of spatial frequencies [in cycles per image, as in Parish and Sperling (1991)] for all stimuli, and this showed higher spatial frequency for words $(\bar{F}=11.9 \pm$
0.4 cycles/image $)$ and Greek strings ( $\bar{F}=9.5 \pm 0.9$ cycles/image $)$ compared with pictures $(\bar{F}=6.9 \pm 4.3$ cycles/image $)$ and nonobjects $(\bar{F}=$ $4.3 \pm 1.4$ cycles/image). During semantic and perceptual decisions, the item above acted as a target that was semantically or physically related to one of the items below, and subjects indicated their responses with a finger press. In the speech production conditions, there was no semantic or perceptual relationship between any of the three items, and subjects were asked to name the three pictures, read the three words, or say " 1,2 , 3 " in response to unfamiliar stimuli. Before each stimulus block, a brief instruction was presented on the screen for $3.6 \mathrm{~s}$ to indicate what sort of response would be necessary. Stimulus presentation in the scanner was via a video projector, a front-projection screen, and a system of mirrors fastened to the MRI head coil. Additional details about the paradigm and stimuli can be found in our previous work (Josse et al., 2008; Seghier et al., 2010).

MRI acquisition. Experiments were performed on a 1.5T Siemens system (Siemens Medical Systems). Functional imaging consisted of an echoplanar imaging with gradient recalled echo sequence $(\mathrm{TR}=3600$ $\mathrm{ms}, \mathrm{TE}=50 \mathrm{~ms}$, flip angle $=90^{\circ}, \mathrm{FOV}=192 \mathrm{~mm}$, matrix $=64 \times 64,40$ axial slices, $2 \mathrm{~mm}$ thick with $1 \mathrm{~mm}$ gap). Anatomical T1-weighted images were acquired using a three-dimensional modified driven equilibrium Fourier transform sequence $(\mathrm{TR}=12.24 \mathrm{~ms}, \mathrm{TE}=3.56 \mathrm{~ms}, \mathrm{TI}=530 \mathrm{~ms}$, matrix $=256 \times 224,176$ sagittal slices with a final resolution of $1 \mathrm{~mm}^{3}$ ).

$f M R I$ data preprocessing. Data processing and statistical analyses were performed with the Statistical Parametric Mapping SPM5 software package (Wellcome Trust Centre for Neuroimaging, London UK). All functional volumes were spatially realigned to the first EPI volume for motion correction, unwarped to correct for artifacts caused by movement-byinhomogeneity interactions, and normalized to the MNI space using the unified normalization-segmentation procedure, with resulting voxels size of $2 \times 2 \times 2 \mathrm{~mm}^{3}$. The normalization to the MNI space was performed by first coregistering the anatomical T1 image to the mean EPI image that was generated during the realignment step, then the unified segmentation was applied to the coregistered anatomical image using the default parameters in SPM5 to estimate the normalization parameters that encode the transformation from the native to MNI space, and finally the normalization parameters were subsequently applied to all realigned EPI images. Note that during the unified segmentation, a symmetrical version of the tissue priors was used (see Symmetrical images, below).

Symmetrical images. During the normalization-segmentation step, we used symmetrical priors created by simply copying, flipping along the $x$-axis, and averaging the original and the mirror (flipped) versions of the default tissue probability maps of SPM5 (Salmond et al., 2000). The resulting normalization-segmentation parameters were then applied to the subject's functional images, thereby rendering them symmetrical, which allows left and right hemisphere activation to be directly compared (Josse et al., 2008; Seghier et al., 2011a). The normalized (symmetrical) functional images were then spatially smoothed with a $6 \mathrm{~mm}$ full-width half-maximum isotropic Gaussian kernel.

First-level analyses. For each individual subject, we performed a fixedeffect analysis on all preprocessed functional volumes of that subject, using the general linear model at each voxel. Time series from each voxel were high-pass filtered ( $1 / 128 \mathrm{~Hz}$ cutoff) to remove low-frequency noise and signal drift. Each stimulus onset was modeled as an event in condition-specific stick-functions with a duration of $4.32 \mathrm{~s}$ per trial and a stimulus onset interval of $4.5 \mathrm{~s}$. The resulting stimulus functions were convolved with a canonical hemodynamic response function that provided regressors for the linear model. The appropriate summary or contrast images were then generated in all subjects for the correct trials of each condition versus fixation.

Voxel-based laterality maps. We generated maps of the laterality difference at each voxel for each subject (Salmond et al., 2000; Liégeois et al., 2002; Nakamura et al., 2005; Xue et al., 2005; Josse et al., 2008; Seghier et al., 2011a). First, the symmetrical contrast images from the first-level analysis were copied and each copy was flipped along the interhemispheric fissure (i.e., $x$-axis mirror images). Then the resulting flipped image was subtracted from its original (unflipped) version to create a laterality map. Voxel-based laterality maps therefore code the difference between each contrast at every voxel and at its homolog in the other 
$y=-54$

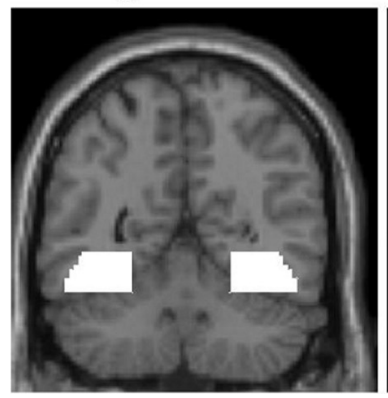

$z=-14$

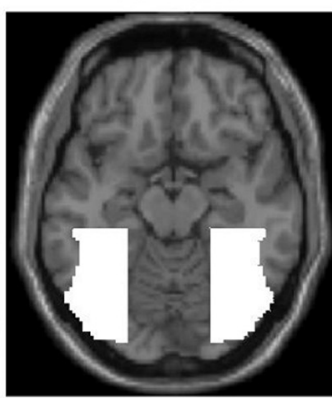

$x=-40$

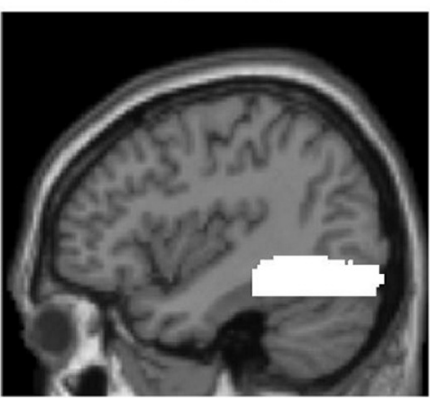

Figure 2. The explicit mask within v0T regions (white) used during the group analysis on laterality maps. This mask was created as follows: (1) AAL atlas (http://www.cyceron.fr/web/aal_anatomical_automatic_labeling.html) was used to delineate the left and right fusiform gyrus; (2) the resulting binary mask was morphologically diluted to ensure including the whole v0T; (3) because all laterality maps were symmetrical, we defined the union between the original binary mask and an $x$-axis mirror of the mask, resulting in a symmetrical mask; and (4) we limited the mask in the posterior-to-anterior direction at $y=-90 \mathrm{~mm}$ and $y=-30$ $\mathrm{mm}$, respectively, and in the inferior-to-superior direction at $z=-20 \mathrm{~mm}$ to $z=-4 \mathrm{~mm}$, respectively.

Table 1. MNI coordinates of lateralized v0T from previous fMRI studies

\begin{tabular}{|c|c|c|}
\hline Study & Lateralized v0T & Task/contrast \\
\hline Cai et al. (2010) & $-46,-46,-16$ & $\begin{array}{l}\text { Lexical decision on words versus checker- } \\
\text { boards }\end{array}$ \\
\hline Cohen et al. (2003) & $\begin{array}{l}-36,-75,-12 \\
-36,-45,-27\end{array}$ & Alphabetic stimuli versus checkerboards \\
\hline $\begin{array}{l}\text { Guo and Burgund } \\
\text { (2010) }\end{array}$ & $\begin{array}{r}49,-57,-10 \\
-41,-66,-10\end{array}$ & $\begin{array}{l}\text { Orthographic, phonological, and semantic } \\
\text { effects on Chinese characters }\end{array}$ \\
\hline Kao et al. (2010) & $-44,-62,-15$ & $\begin{array}{l}\text { Chinese characters contrasted with their } \\
\text { inverted versions }\end{array}$ \\
\hline $\begin{array}{l}\text { Nakamura et al. } \\
\text { (2005) }\end{array}$ & $-42,-67,-17$ & $\begin{array}{l}\text { Kanji and Kana characters relative to } \\
\text { fixation (using laterality maps) }\end{array}$ \\
\hline $\begin{array}{l}\text { Pinel and Dehaene } \\
\text { (2010) }\end{array}$ & $-45,-56,-10$ & $\begin{array}{l}\text { Sentence reading versus checkerboards } \\
\text { (using laterality maps) }\end{array}$ \\
\hline Sabsevitz et al. (2005) & $-45,-52,-15$ & Concrete versus abstract words \\
\hline Szwed et al. (2011) & $\begin{array}{l}-45,-41,-18 \\
\text { varying from } y=-54 \\
\quad \text { to } y=-80\end{array}$ & $\begin{array}{l}\text { Words versus scrambled control stimuli } \\
\text { (using laterality maps) }\end{array}$ \\
\hline Vigneau et al. (2005) & $\begin{array}{l}-44,-76,-14 \\
-46,-40,-12\end{array}$ & Word reading relative to fixation \\
\hline $\begin{array}{l}\text { Woodhead et al. } \\
\text { (2011) }\end{array}$ & $-40,-55,-13$ & $\begin{array}{l}\text { Words versus scrambled stimuli, and high } \\
\text { versus low spatial frequency stimuli }\end{array}$ \\
\hline Xue et al. (2005) & $-39,-68,-12$ & $\begin{array}{l}\text { Phonological/semantic tasks on Chinese } \\
\text { words relative to fixation (using later- } \\
\text { ality maps) }\end{array}$ \\
\hline
\end{tabular}

hemisphere. Thus, they code the interaction (Liégeois et al., 2002) between task (activation vs control) and hemisphere (left vs right) at each voxel. Our voxel-based maps were based on subtraction between left $(\mathrm{L})$ and right $(\mathrm{R})$ hemisphere activation at each voxel (i.e., $\mathrm{L}-\mathrm{R}$ ) rather than a relative difference scaled to total signal at that voxel (i.e., as a laterality index: $\mathrm{L}-\mathrm{R} / \mathrm{L}+\mathrm{R}$ ). Although a relative difference is suitable for correcting global differences in hemispheric activation, it is not recommended at the voxel level because it may introduce nonlinearities in the relationship between left and right signal. For example, the same difference between left and right $(\mathrm{L}-\mathrm{R})$ would have a smaller relative difference in a highly activated voxel (i.e., $L+R$ very large) than a weakly activated voxel. The method of choice for voxel-based lateralization at the group level was therefore based on subtraction between left and right rather than their relative difference (Liégeois et al., 2002; Xue et al., 2005; Baciu et al., 2005; Nakamura et al., 2005; Cousin et al., 2007; Josse et al., 2008; Pinel and Dehaene, 2010; Szwed et al., 2011).

Voxel-based second-level group analyses. We conducted second-level ANOVA analysis on either original (unflipped) contrast images or voxelbased laterality maps. All eight contrasts were included in this analysis and group effects were assessed within an explicit mask that contained the whole ventral occipitotemporal cortex in each hemisphere (Fig. 2). The analysis on the voxel-based laterality maps identified the voxels with the most consistently lateralized activation for any condition in our 82 subjects (Liégeois et al., 2002). All effects are reported at FWE-corrected level of $p<0.05$. In addition, the consistency across subjects of lateralized voxels was examined in each individual laterality map and was defined as the number of subjects with "left minus right activity" in the same direction (sign) as the group effect.

Predictions. Our predictions were as follows: if left lateralized vOT responses for words are related to: (1) visual factors (Rossion et al., 2003; Hsiao and Cottrell, 2009; Woodhead et al., 2011) then activation should also be left lateralized for other stimuli with similar visual features (i.e., meaningless Greek letter strings); (2) visual word form selectivity in left vOT (Cohen et al., 2003; Dehaene and Cohen, 2007; Vinckier et al., 2007; Szwed et al., 2011), then left vOT activation for words should be increased relative to all other stimuli including pictures of familiar objects; and (3) semantic processing, then left laterality should be stronger for words and objects than unfamiliar Greek letters and nonobjects (Sabsevitz et al., 2005; Vigneau et al., 2005). Moreover, we expect the influence of these factors to interact with task (Nakamura et al., 2005; Vigneau et al., 2005; Large et al., 2007; Guo and Burgund, 2010; Wang et al., 2011) and vary with the subregions of vOT tested because previous studies have shown differences in functional responses and connectivity in posterior and anterior parts of vOT (Moore and Price, 1999; Mechelli et al., 2005; Price and Mechelli, 2005; Vinckier et al., 2007; Seghier et al., 2008; Seghier and Price, 2010; Szwed et al., 2011; Wang et al., 2011). Furthermore, our motivation for mapping laterality at the voxel level is to visualize the different spatial profiles at high definition because the exact location of literalized vOT varied considerably across previous fMRI studies (Table 1).

\section{Results}

\section{Behavioral responses}

All subjects performed all tasks with high accuracy (>80\%). In the semantic matching conditions, the mean response times were comparable between words and pictures $(1.69 \pm 0.3 \mathrm{~s}$ and $1.72 \pm$ $0.3 \mathrm{~s}$, respectively), with no significant difference $(t=1.3, p>$ 0.1 ). We were unable to extract response times for the naming and reading conditions from the speech production recordings, but note that the fast presentation rate ensured that correct naming response times were always $<1.4 \mathrm{~s}$ and that the interpretation of our data does not depend on significant latency differences between words and pictures (see Consistencies across participants, below).

\section{Lateralization for words dissociated three vOT subdivisions}

The dependency of vOT activation on task, stimuli, and hemisphere is illustrated in Figure 3. For words, activation was strongly left lateralized (at $p<0.05$, FWE-corrected), regardless of task (reading aloud or semantic decisions) or baseline (fixation, Greek letters, pictures of objects, or pictures of nonobjects) (Figs. 3, first and fifth columns; 4) . Specifically, strong left lateralization was observed in three subdivisions (Fig. $4 A$ ): posterior vOT $(x=-42, y=-70, z=-10)$, middle vOT (global peak at $-44,-54,-16$; second peak at $-44,-62,-14)$, and anterior vOT $(-44,-44,-14)$, with high consistency across the 82 subjects (Fig. $4 \mathrm{~B}$ ). The three left vOT peaks were identified according to their particular lateralization profiles across tasks and stimuli, 


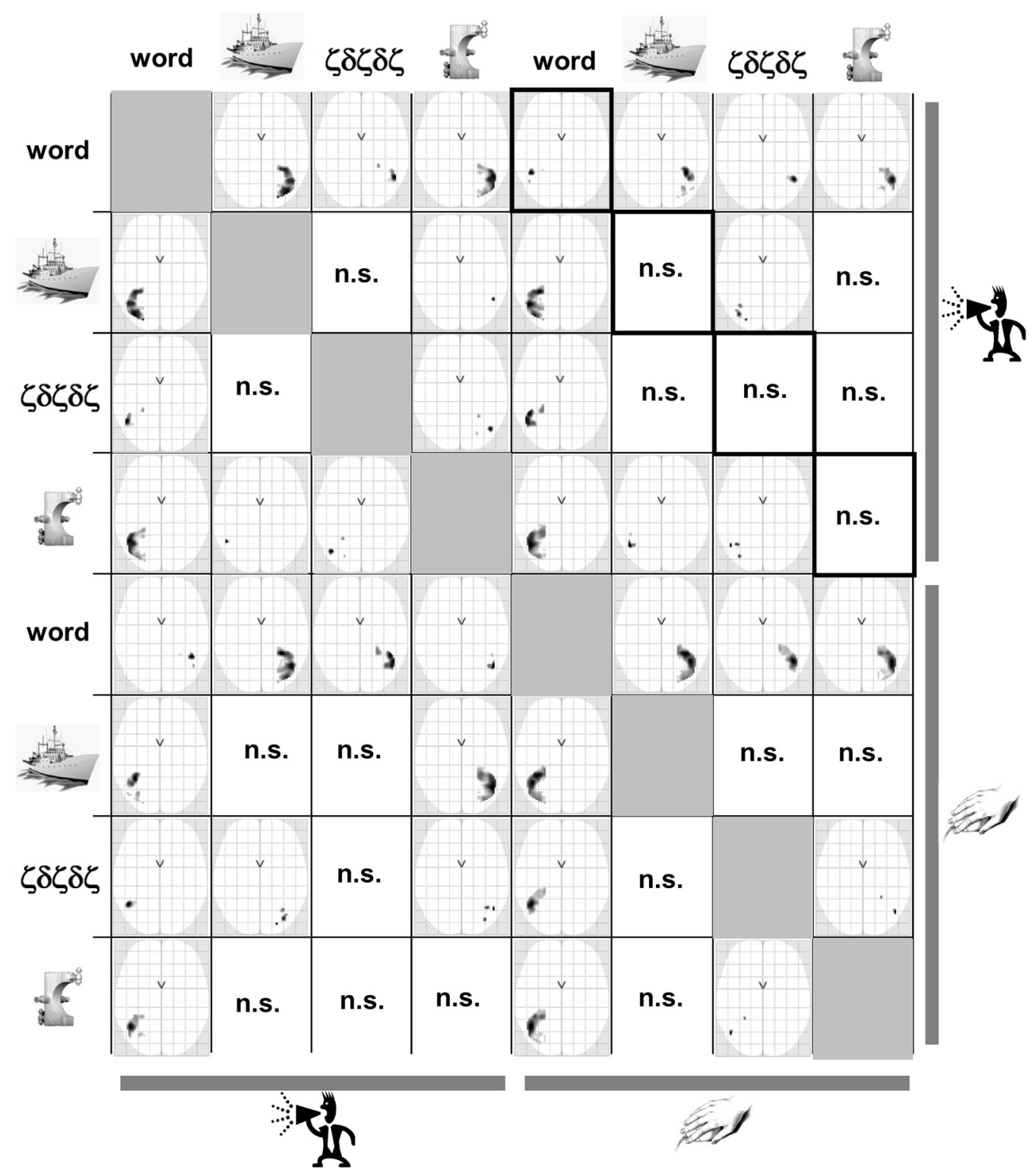

Figure 3. The full eight-by-eight pairwise comparisons of laterality maps across tasks and stimuli. This figure represents the interaction between activation and hemisphere. Each column represents the comparison between laterality in a given condition and laterality in each condition of the remaining conditions (e.g., the first column represents the comparison of laterality for words during reading with laterality during the other conditions). Thick black outlines represent the task effect on laterality when stimuli were held constant. Note that by construction, this matrix is skew-symmetric (i.e., antimetric). n.s., Not significant at $p<0.05$, FWE-corrected.

with posterior vOT being the global maximum of lateralization for letter stimuli (words and Greek letters) relative to fixation, middle vOT being the global maximum of lateralization for words relative to all other stimuli regardless of task, and anterior vOT being the global maximum of lateralization for semantic decisions on words relative to reading aloud and fixation. We considered how lateralization in each vOT subdivision was explained by the relative contribution of the left or right hemisphere activation.

\section{Left vOT lateralization for words results from reduced right vOT involvement}

Critically, increased left lateralization for words relative to nonverbal stimuli was not indicative of greater left hemisphere activation for words. To the contrary, activation was less for words than for pictures of objects in all three vOT regions (Fig. 5) in both the left and right hemispheres. The important point here is that the reason vOT activation was more strongly left lateralized for words than for pictures of objects is that the reduction in activation for words relative to objects was greater in right than in left vOT regions. Furthermore, when we assessed the correlation across subjects (Seghier et al., 2008) between activation in each vOT region and its right homolog, we found that left and right hemisphere activation covaried significantly across subjects for all stimuli and conditions except words $(p>0.05$; Table 2). This suggests that activation in right vOT regions was disengaged from that in the homolog left vOT regions for words but not during the processing of pictures or unfamiliar Greek letters. Below, we considered how activation and lateralization varied with task and stimuli in each subregion.

\section{Left lateralization in posterior vOT is related to visual processing}

In posterior vOT, activation was left lateralized for meaningless Greek letters and words relative to fixation (Fig. 4) but not for 


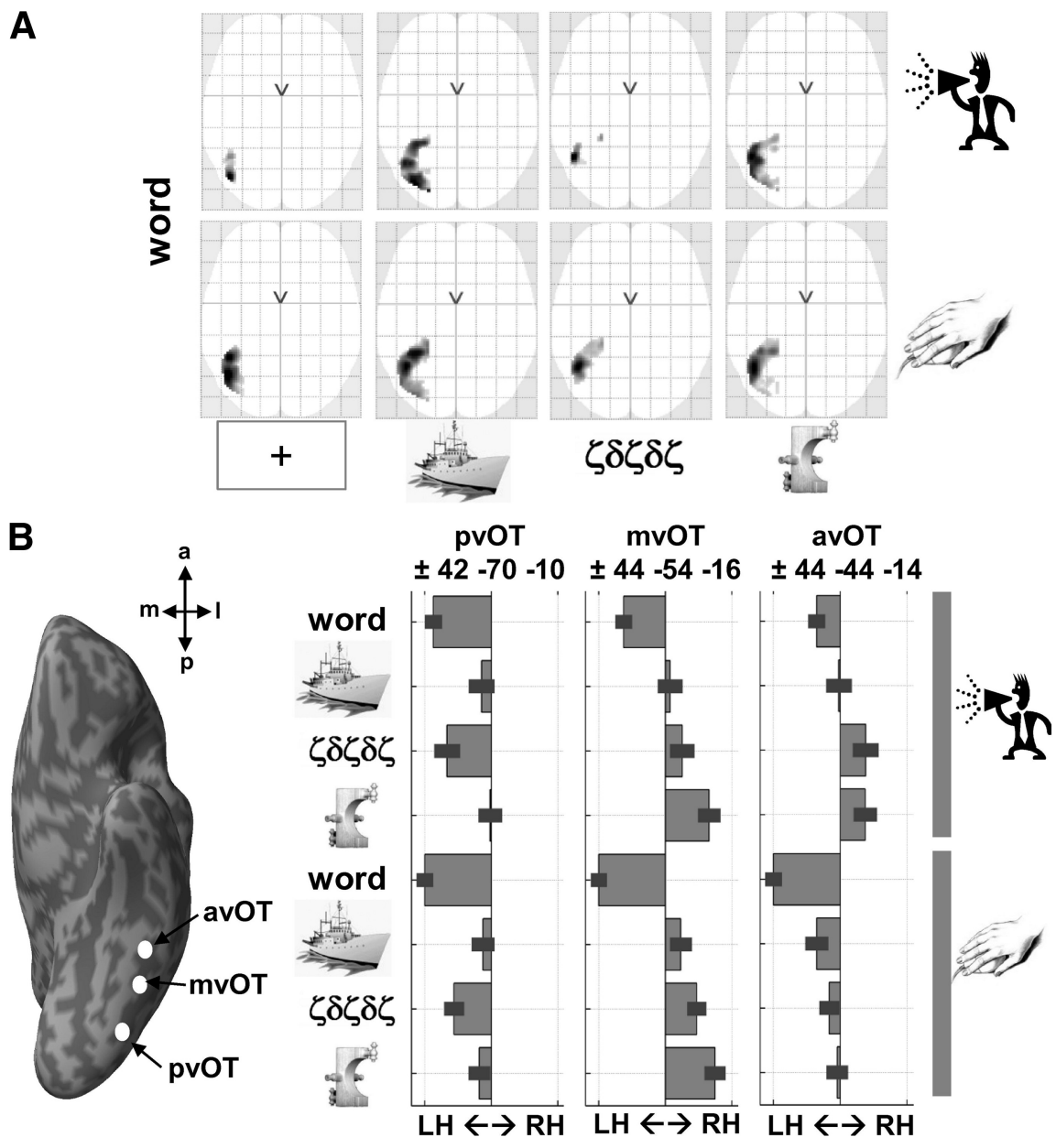

Figure 4. $\quad A$, Consistent effect of laterality for words at the voxel level (at $p<0.05$, FWE-corrected over 82 subjects), shown on axial view of the glass brain of SPM. Strong left laterality was identified for words during both tasks (production and matching) relative to fixation, pictures of familiar objects, unfamiliar Greek letters, and pictures of unfamiliar nonobjects. $\boldsymbol{B}$, The relative difference in signal (average \pm SD over 82 subjects) between left and right activity in our three v0T regions (coordinates shown on top of each bar graph), with bars on the left side indicating left laterality and bars on the right indicating right laterality. pvOT, Posterior v0T; mv0T, middle v0T; av0T, anterior v0T; LH, left hemisphere; RH, right hemisphere.

objects $(Z=2.0)$. These findings cannot be explained by visual attributes because neither the left or right anterior vOT areas were consistently activated by all visual stimuli. We propose that lateralization in anterior vOT is a simple consequence of semantic processing in the left hemisphere because it was (1) greater for meaningful stimuli (objects and words) than unfamiliar stimuli (nonobjects and Greek letters) in both tasks and (2) greater for semantic decisions on words than reading aloud, with (3) no significant difference between semantic decisions on objects or words despite the contrasting visual attributes of these stimuli. Moreover, this is not due to the matching per se, because the difference in activation for perceptual matching relative to saying " $1,2,3$ " on unfamiliar stimuli was located in medial anterior fusiform (at $p<0.05$, FWEcorrected), not the more lateral anterior vOT. However, the difference in left anterior vOT between words and objects was significant during the naming/reading tasks. This task-by-stimulus interaction was a consequence of left vOT activation being lower during reading than picture naming, semantic decisions on words, or semantic decisions on pictures. It is consistent with left vOT being a semantic processing region and with cognitive models of naming and reading in which picture naming is more reliant on semantic mediation than reading (Glaser and Glaser, 1989).

\section{Left lateralization in middle vOT reflects a combination of visual and} nonvisual factors

In middle vOT, activation was left lateral-

pictures of objects or unfamiliar nonobjects (Fig. 5). As observed for words, left lateralization for Greek letters was the consequence of a greater reduction in right than left posterior vOT activation for letter stimuli than picture stimuli. As the Greek letter strings were unfamiliar and meaningless, left lateralized responses cannot be explained by linguistic processing, visual expertise, or recognizability. It is therefore likely that activation in left and right posterior vOT was related to visual attributes, consistent with other observations that posterior vOT was significantly activated by all visual stimuli relative to fixation, with higher activation for pictures of objects and nonobjects (Figs. 3, 5). Accordingly, we propose that the strong left lateralization in posterior vOT is driven by the reduced activation in right posterior vOT for letters and words relative to pictures of objects and nonobjects. This might be a consequence of our letter and word stimuli having less information in the low spatial frequency band (see Materials and Methods, above).

\section{Left lateralization in anterior vOT is related to semantic processing}

In anterior vOT, left lateralization for words was stronger during semantic decisions than reading aloud $(Z=5.1$; Fig. 3, fifth column). There was a similar but nonsignificant effect of task on ized for words relative to Greek letters and fixation, and left lateralized for objects relative to nonobjects (Fig. 3), but not lateralized for objects relative to fixation, and right lateralized for unfamiliar nonobjects relative to fixation $(p<0.05$, FWEcorrected). By considering the response of each hemisphere in turn, we suggest that lateralization in middle vOT reflects a combination of visual expertise in right middle vOT and semantics in left middle vOT. We associate right middle vOT activation with visual expertise (less activation with more expertise) because it was higher for unfamiliar Greek letters than words that were matched for spatial frequency and visual complexity and not significantly different for nonobjects and Greek letters that vary in spatial frequency and visual complexity (Fig. 5). We associate left middle vOT activation with semantics because it was stronger for objects and words than meaningless nonobjects and Greek letters, and semantic decisions on words relative to reading aloud.

\section{Consistencies across participants}

The degree to which activation was left lateralization for word processing was not significantly related ( $p>0.05$, corrected) to differences in scanner performance, response times, or demographic variables (age, gender, and handedness) in any of the vOT subdivisions. Specifically, of 44 right-handers and 38 left- 

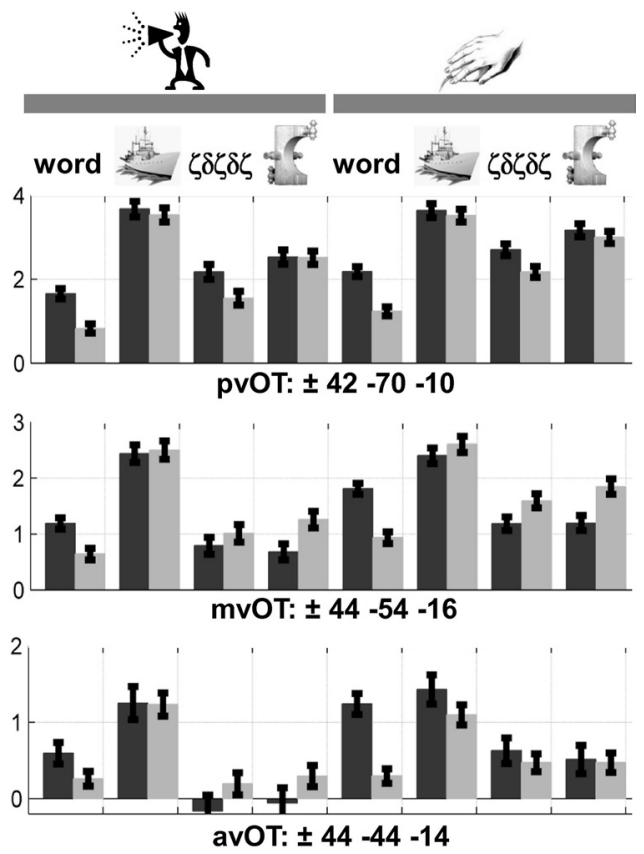

Figure 5. Mean signal level over our 82 subjects in left (dark gray) and right (light gray) hemisphere of each v0T region in our eight conditions relative to fixation. pv0T, Posterior v0T; mv0T, middle v0T; avOT, anterior v0T.

Table 2. Correlations between left and right activity of each v0T region during the eight conditions

\begin{tabular}{|c|c|c|c|}
\hline \multirow[b]{2}{*}{ Conditions } & \multicolumn{3}{|l|}{ Correlations } \\
\hline & $\begin{array}{l}\text { Left/right } \\
\text { posterior v0T }\end{array}$ & $\begin{array}{l}\text { Left/right } \\
\text { middle v0T }\end{array}$ & $\begin{array}{l}\text { Left/right } \\
\text { anterior v0T }\end{array}$ \\
\hline \multirow[t]{2}{*}{ Reading: words } & $r=0.15$ & $r=0.22$ & $r=0.33$ \\
\hline & $p>0.1$ & $p>0.05$ & $p=0.002$ \\
\hline \multirow[t]{2}{*}{ Naming: pictures } & $r=0.63$ & $r=0.33$ & $r=0.25$ \\
\hline & $p<0.001$ & $p=0.002$ & $p=0.02$ \\
\hline \multirow[t]{2}{*}{ Say "1, 2, 3": symbols } & $r=0.44$ & $r=0.38$ & $r=0.23$ \\
\hline & $p<0.001$ & $p<0.001$ & $p=0.05$ \\
\hline \multirow[t]{2}{*}{ Say " $1,2,3$ ": nonobjects } & $r=0.54$ & $r=0.45$ & $r=0.37$ \\
\hline & $p<0.001$ & $p<0.001$ & $p<0.001$ \\
\hline \multirow[t]{2}{*}{ Semantic matching: words } & $r=0.48$ & $r=0.18$ & $r=0.08$ \\
\hline & $p<0.001$ & $p>0.1$ & $p>0.1$ \\
\hline \multirow[t]{2}{*}{ Semantic matching: pictures } & $r=0.46$ & $r=0.37$ & $r=0.33$ \\
\hline & $p<0.001$ & $p<0.001$ & $p=0.002$ \\
\hline \multirow[t]{2}{*}{ Perceptual matching: symbols } & $r=0.50$ & $r=0.46$ & $r=0.27$ \\
\hline & $p<0.001$ & $p<0.001$ & $p=0.01$ \\
\hline \multirow[t]{2}{*}{ Perceptual matching: nonobjects } & $r=0.44$ & $r=0.43$ & $r=0.30$ \\
\hline & $p<0.001$ & $p<0.001$ & $p=0.006$ \\
\hline
\end{tabular}

The correlations were assessed across all our 82 subjects. Boldface represents correlations that are not significant at $p<0.05$, uncorrected.

handers, during semantic matching on words relative to fixation, left lateralization was observed in 37,44 , and 42 right-handers and in 35,33 , and 36 left-handers in posterior, middle, and anterior vOT, respectively. During reading aloud relative to fixation, left lateralization was observed in 37,39 , and 37 right-handers and in 32, 32, and 30 left-handers in posterior, middle, and anterior vOT, respectively. Thus, the majority (80-100\%) of our right and non-right-handers show higher responses in left than right vOT during visual word processing.

Right lateralization in medial regions was strong for all stimuli except words

Medial to our three vOT subdivisions, we observed strong right lateralization ( $p<0.05$, FWE corrected) in the posterior fusiform at $34,-72,-18$ for pictures of objects and nonobjects that have low spatial frequency but not for words or Greek letter strings that have high spatial frequency (Fig. 6). A second rightlateralized cluster was identified in anterior fusiform at +28 , $-42,-18$ for all stimuli except words (objects, Greek letters, and nonobjects; Fig. 6). This right anterior medial fusiform cluster is remarkably similar to the region where Nakamura et al. (2005) observed right lateralization for pictures of objects and logograms relative to fixation but not for words relative to fixation. Right lateralization for pictures of familiar objects was also highly consistent across our 82 subjects and was observed in 32 and 41 right-handers and in 33 and 35 left-handers in posterior and anterior medial fusiform regions, respectively, during object naming, and in 33 and 37 right-handers and in 31 and 37 lefthanders in posterior and anterior medial fusiform regions, respectively, during semantic decisions on pictures. Thus, the majority (80-97\%) of our right- and non-right-handers show higher responses in right than left medial fusiform during object processing.

\section{Discussion}

In this study, we investigated whether the well characterized leftlateralized signature for written words was best explained in terms of visual attributes of the stimuli, specialization for visual word form processing, and/or semantic processing. We found that the best explanation of the data depended on which subdivision of vOT was being tested. As discussed in detail below, lateralization in posterior vOT was best explained by visual processing, lateralization in anterior vOT was best explained by semantic processing, and lateralization in middle vOT was best explained by a combination of visual processing in the right hemisphere and the semantic demands of the task in the left hemisphere. These region-specific findings allow us to validate and integrate previously conflicting interpretations of lateralized activation in vOT.

In addition, our results show that it is necessary to consider the right hemisphere contribution to vOT lateralization because we found that the degree to which vOT activation was left lateralized was a consequence of reduced right hemisphere activation rather than increased left hemisphere activation. This is not consistent with left hemisphere specialization for words, but it does suggest that reading expertise reduces the need for right hemisphere visual processing. Below, we discuss the data supporting our conclusions in each subregion, and the implications of our findings for models of reading and previous accounts of functional laterality in vOT (Rossion et al., 2003; Nakamura et al., 2005; Vigneau et al., 2005; Xue et al., 2006; Maurer and McCandliss, 2007; Cai et al., 2008, 2010; Maurer et al., 2008; Hsiao and Cottrell, 2009; Kao et al., 2010; Pinel and Dehaene, 2010; Szwed et al., 2011; Twomey et al., 2011).

In the posterior vOT region, activation was left lateralized for words and Greek letters that had high spatial frequency, but there was no significant lateralization for photographs of objects and nonobjects that had lower spatial frequency. The degree to which posterior vOT activation was left lateralized was not related to recognizability (words and pictures vs letters and nonobjects) or task. We therefore conclude that lateralization in posterior vOT is driven by visual-feature processing (Rossion et al., 2003). Previous fMRI studies have also reported that, when the baseline is fixation, activation is left lateralized in posterior vOT for stimuli with high spatial frequency (Nakamura et al., 2005; Vigneau et al., 2005), including words, logograms, nonwords and line drawings of objects (which have higher spatial frequency than the grayscale photographs of objects and nonobjects that we used here). Our 


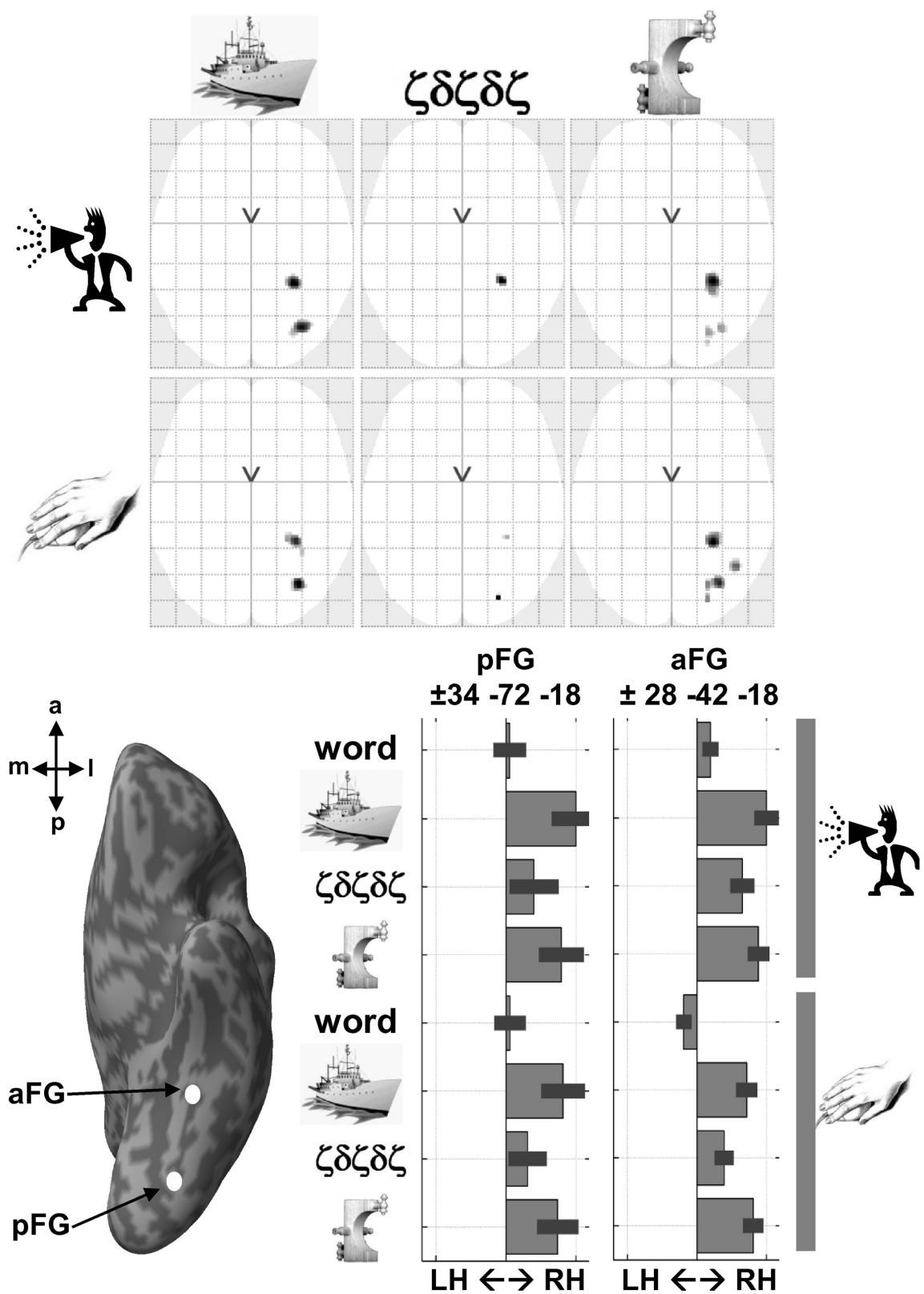

Figure 6. Top, Consistent effect of laterality at the voxel level (at $p<0.05$, FWE-corrected over 82 subjects) for all other stimuli (pictures of familiar objects, unfamiliar Greek symbols, and pictures of unfamiliar nonobjects) relative to fixation, shown on axial view of the glass brain of SPM. Bottom, The relative difference in signal (average \pm SD over 82 subjects) between left and right activity is illustrated in the two right-lateralized fusiform regions (coordinates shown on top of each bar graph), with bars on the left side indicating a stronger left laterality and bars on the right indicating a stronger right laterality. pFG, Posterior fusiform gyrus; aFG, anterior fusiform gyrus; $\mathrm{LH}$, left hemisphere; $\mathrm{RH}$, right hemisphere.

findings in posterior vOT are therefore consistent with prior evidence that the left and right occipitotemporal cortices are differentially sensitive to high versus low spatial frequency inputs (Han et al., 2002; Peyrin et al., 2004; Mercure et al., 2008; Woodhead et al., 2011). Note that the causes of left lateralization in posterior vOT that we observed here for English words may vary for other logographic scripts (Bolger et al., 2005) such as Chinese (Peng et al., 2004; Xue et al., 2005), Kanji (Uchida et al., 1999; Koyama et al., 2011), or Hangul (Lee, 2004) characters. For instance, strong left lateralization in posterior vOT at $-39,-68,-12$ was suggested to be linked to language factors rather than to visual features of Chinese words (Xue et al., 2005).
A very different pattern of effects was observed in anterior vOT. Here, activation was left lateralized during semantic decisions on words with a trend on pictures of objects but not during either meaningless Greek letters or nonobjects. The semantic nature of the left lateralization in anterior vOT was further supported by increased left anterior vOT activation for semantic matching on words relative to reading aloud and for both words and pictures relative to perceptual matching on unfamiliar stimuli (Seghier et al., 2010, their Fig. 1; 2011b, their Fig. 1). Moreover, the association of left anterior vOT with semantics is consistent with previous studies showing that left anterior vOT is involved in semantic more than phonological processing of both visual and auditory stimuli (Mummery et al., 1998; Binder et al., 2009; Davis and Gaskell, 2009; Sharp et al., 2010). However, this does not rule out a potential semantic contribution of the right hemisphere (Lambon Ralph and Patterson, 2008; Lambon Ralph et al., 2010) that may impact on vOT laterality (Sabsevitz et al., 2005).

A third pattern of effects was observed in the middle vOT region. Relative to fixation, left lateralization was only observed for words, which might be interpreted as a word-selective effect. Contrary to this conclusion, however, left middle vOT activation was less for words than pictures during both tasks, with higher left lateralization for words resulting from an even greater reduction in right middle vOT activation for words relative to pictures or other unfamiliar stimuli. Moreover, activation in middle vOT became left lateralized for pictures of objects when the baseline was nonobjects rather than fixation. Therefore, by partially controlling for visual-feature processing, the nonobject baseline unveiled left lateralized activation for meaningful stimuli.

The reduced activation in right middle vOT for words was not related to visual complexity or spatial frequency (as in posterior vOT) because right middle vOT activation was the same for unfamiliar Greek letters and nonobjects that clearly differed in their visual complexity. However, reduced right middle vOT activation for words relative to unfamiliar Greek letters can be explained in terms of visual expertise with the visual features of the stimuli. We therefore suggest that lateralization in middle vOT depends on a combination of visual expertise in the right hemisphere and semantics in the left hemisphere.

The implication for models of reading is that left lateralized vOT activation for words relative to other stimuli should not be interpreted as increased word-specific processing in left vOT. Instead, we have shown that the unique signature for words in the 
ventral visual stream results from low activation in right vOT regions (Fig. 4) and negligible activation in medial fusiform regions (Fig. 6). Reduced right hemisphere activation in these regions for words might be a consequence of less reliance on visual feature processing in the context of greater top-down support from the language system. This hypothesis is consistent with the interactive view of vOT function that proposes that vOT integrates top-down predictions from the language system with bottom-up visual inputs (Devlin et al., 2006; Kherif et al., 2011; Price and Devlin, 2011; Twomey et al., 2011). The current study was not designed to elucidate the source of the top-down effects. This will require future studies that use high-temporal resolution techniques to show, for instance, activation in temporofrontal regions at a latency similar to or even earlier than vOT during word processing (Pammer et al., 2004; Mainy et al., 2008; Cornelissen et al., 2009).

Our findings also have multiple implications for previous explanations of left lateralization in vOT for words. First, the spatial frequency account (Hsiao and Cottrell, 2009; Woodhead et al., 2011) may explain lateralization in posterior vOT regions but not in middle or anterior vOT. Second, the extensive left-lateralized pattern in vOT for words we observed here is in line with that reported in Szwed et al. (2011) for words relative to scrambled stimuli; however, our results also show that (1) this pattern persisted even when compared with other control conditions (i.e., fixation, objects, and unfamiliar stimuli); (2) its determinants varied from posterior to anterior vOT regions, with the impact of language increasing along the posterior-to-anterior dimension; and (3) laterality was task-dependent in anterior vOT (Fig. 3), which illustrates that left lateralization for words is not only determined by how we see words but also by what we do with words. The combined influences from stimulus properties, task, familiarity, expertise, and the verbal content of stimuli may also explain why activation is left lateralized for words in skilled readers and right lateralized in kindergarten children who have not yet learnt to link print to sound (Maurer et al., 2006, 2010). Future work is needed to identify whether the visual and nonvisual factors that influence the determinants of vOT laterality depend on the script and language being tested (Bolger et al., 2005; Xue et al., 2005; Hellige and Adamson, 2006; Maurer et al., 2008; Wong et al., 2009).

In summary, in a large group of 82 skilled readers, we have characterized different patterns of lateralization in three different subregions of the ventral occipital temporal cortex and discussed the potential underlying mechanisms. By showing that different mechanisms explain lateralization in different vOT regions, we are able to integrate previously conflicting results. In addition, we also highlight the strong contribution of right vOT activation to left lateralization during reading. These findings have implications for understanding the neural basis of reading in neurologically normal participants and patients with left or right vOT damage. They also have implications for studies that used laterality as a marker for efficient (Xue et al., 2006) or impaired (Abrams et al., 2009) reading.

\section{References}

Abrams DA, Nicol T, Zecker S, Kraus N (2009) Abnormal cortical processing of the syllable rate of speech in poor readers. J Neurosci 29:7686-7693.

Baciu M, Juphard A, Cousin E, Bas JF (2005) Evaluating fMRI methods for assessing hemispheric language dominance in healthy subjects. Eur J Radiol 55:209-218.

Baker CI, Liu J, Wald LL, Kwong KK, Benner T, Kanwisher N (2007) Visual word processing and experiential origins of functional selectivity in human extrastriate cortex. Proc Natl Acad Sci U S A 104:9087-9092.
Binder JR, Desai RH, Graves WW, Conant LL (2009) Where is the semantic system? A critical review and meta-analysis of 120 functional neuroimaging studies. Cereb Cortex 19:2767-2796.

Bolger DJ, Perfetti CA, Schneider W (2005) Cross-cultural effect on the brain revisited: universal structures plus writing system variation. Hum Brain Mapp 25:92-104.

Cai Q, Lavidor M, Brysbaert M, Paulignan Y, Nazir TA (2008) Cerebral lateralization of frontal lobe language processes and lateralization of the posterior visual word processing system. J Cogn Neurosci 20:672-681.

Cai Q, Paulignan Y, Brysbaert M, Ibarrola D, Nazir TA (2010) The left ventral occipito-temporal response to words depends on language lateralization but not on visual familiarity. Cereb Cortex 20:1153-1163.

Cohen L, Martinaud O, Lemer C, Lehéricy S, Samson Y, Obadia M, Slachevsky A, Dehaene S (2003) Visual word recognition in the left and right hemispheres: anatomical and functional correlates of peripheral alexias. Cereb Cortex 13:1313-1333.

Cornelissen PL, Kringelbach ML, Ellis AW, Whitney C, Holliday IE, Hansen PC (2009) Activation of the left inferior frontal gyrus in the first $200 \mathrm{~ms}$ of reading: evidence from magnetoencephalography (MEG). PloS One 4:e5359.

Cousin E, Peyrin C, Pichat C, Lamalle L, Le Bas JF, Baciu M (2007) Functional MRI approach for assessing hemispheric predominance of regions activated by a phonological and a semantic task. Eur J Radiol 63:274-285.

Davis MH, Gaskell MG (2009) A complementary systems account of word learning: neural and behavioural evidence. Philos Trans R Soc Lond B Biol Sci 364:3773-3800.

Dehaene S, Cohen L (2007) Cultural recycling of cortical maps. Neuron 56:384-398.

Devlin JT, Jamison HL, Gonnerman LM, Matthews PM (2006) The role of the posterior fusiform gyrus in reading. J Cogn Neurosci 18:911-922.

Gauthier I, Tarr MJ, Anderson AW, Skudlarski P, Gore JC (1999) Activation of the middle fusiform 'face area' increases with expertise in recognizing novel objects. Nat Neurosci 2:568-573.

Gauthier I, Skudlarski P, Gore JC, Anderson AW (2000) Expertise for cars and birds recruits brain areas involved in face recognition. Nat Neurosci 3:191-197.

Glaser WR, Glaser MO (1989) Context effects in stroop-like word and picture processing. J Exp Psychol Gen 118:13-42.

Guo Y, Burgund ED (2010) Task effects in the mid-fusiform gyrus: a comparison of orthographic, phonological, and semantic processing of Chinese characters. Brain Lang 115:113-120.

Han S, Weaver JA, Murray SO, Kang X, Yund EW, Woods DL (2002) Hemispheric asymmetry in global/local processing: effects of stimulus position and spatial frequency. Neuroimage 17:1290-1299.

Hellige JB, Adamson MM (2006) Laterality across the world's languages. In: Encyclopedia of language and linguistics, 2nd Edition (Brown K, ed), pp 709-719. Oxford: Elsevier.

Hsiao JH, Cottrell GW (2009) What is the cause of left hemisphere lateralization of English visual word recognition? Pre-existing language lateralization, or task characteristics? Paper presented at the 31st Annual Meeting of the Cognitive Science Society, Amsterdam, the Netherlands, July.

Hunter ZR, Brysbaert M, Knecht S (2007) Foveal word reading requires interhemispheric communication. J Cogn Neurosci 19:1373-1387.

Josse G, Seghier ML, Kherif F, Price CJ (2008) Explaining function with anatomy: language lateralization and corpus callosum size. J Neurosci 28:14132-14139.

Kao CH, Chen DY, Chen CC (2010) The inversion effect in visual word form processing. Cortex 46:217-230.

Kherif F, Josse G, Price CJ (2011) Automatic top-down processing explains common left occipito-temporal responses to visual words and objects. Cereb Cortex 21:103-114.

Koyama MS, Stein JF, Stoodley CJ, Hansen PC (2011) Functional MRI evidence for the importance of visual short-term memory in logographic reading. Eur J Neurosci 33:539-548.

Laeng B, Rouw R (2001) Canonical views of faces and the cerebral hemispheres. Laterality 6:193-224.

Lambon Ralph MA, Patterson K (2008) Generalization and differentiation in semantic memory: insights from semantic dementia. Ann N Y Acad Sci 1124:61-76.

Lambon Ralph MA, Cipolotti L, Manes F, Patterson K (2010) Taking both 
sides: do unilateral anterior temporal lobe lesions disrupt semantic memory? Brain 133:3243-3255.

Large ME, Aldcroft A, Vilis T (2007) Task-related laterality effects in the lateral occipital complex. Brain Res 1128:130-138.

Lee KM (2004) Functional MRI comparison between reading ideographic and phonographic scripts of one language. Brain Lang 91:245-251.

Liégeois F, Connelly A, Salmond CH, Gadian DG, Vargha-Khadem F, Baldeweg T (2002) A direct test for lateralization of language activation using fMRI: comparison with invasive assessments in children with epilepsy. Neuroimage 17:1861-1867.

Mainy N, Jung J, Baciu M, Kahane P, Schoendorff B, Minotti L, Hoffmann D, Bertrand O, Lachaux JP (2008) Cortical dynamics of word recognition. Hum Brain Mapp 29:1215-1230.

Maurer U, McCandliss BD (2007) The development of visual expertise for words: the contribution of electrophysiology. In: Single-word reading: behavioral and biological perspectives (Grigorenko EL, Naples AJ, eds), pp 43-64. New York: Lawrence Erlbaum Associates.

Maurer U, Brem S, Kranz F, Bucher K, Benz R, Halder P, Steinhausen HC, Brandeis D (2006) Coarse neural tuning for print peaks when children learn to read. Neuroimage 33:749-758.

Maurer U, Zevin JD, McCandliss BD (2008) Left-lateralized N170 effects of visual expertise in reading: evidence from Japanese syllabic and logographic scripts. J Cogn Neurosci 20:1878-1891.

Maurer U, Blau VC, Yoncheva YN, McCandliss BD (2010) Development of visual expertise for reading: rapid emergence of visual familiarity for an artificial script. Dev Neuropsychol 35:404-422.

Mechelli A, Crinion JT, Long S, Friston KJ, Lambon Ralph MA, Patterson K, McClelland JL, Price CJ (2005) Dissociating reading processes on the basis of neuronal interactions. J Cogn Neurosci 17:1753-1765.

Mercure E, Dick F, Halit H, Kaufman J, Johnson MH (2008) Differential lateralization for words and faces: category or psychophysics? J Cogn Neurosci 20:2070-2087.

Moore CJ, Price CJ (1999) Three distinct ventral occipitotemporal regions for reading and object naming. Neuroimage 10:181-192.

Mummery CJ, Patterson K, Hodges JR, Price CJ (1998) Functional neuroanatomy of the semantic system: divisible by what? J Cogn Neurosci 10:766-777.

Nakamura K, Oga T, Okada T, Sadato N, Takayama Y, Wydell T, Yonekura Y, Fukuyama H (2005) Hemispheric asymmetry emerges at distinct parts of the occipitotemporal cortex for objects, logograms and phonograms: a functional MRI study. Neuroimage 28:521-528.

Pammer K, Hansen PC, Kringelbach ML, Holliday I, Barnes G, Hillebrand A, Singh KD, Cornelissen PL (2004) Visual word recognition: the first half second. Neuroimage 22:1819-1825.

Parish DH, Sperling G (1991) Object spatial frequencies, retinal spatial frequencies, noise, and the efficiency of letter discrimination. Vision Res 31:1399-1415.

Peng DL, Ding GS, Perry C, Xu D, Jin Z, Luo Q, Zhang L, Deng Y (2004) fMRI evidence for the automatic phonological activation of briefly presented words. Brain Res Cogn Brain Res 20:156-164.

Peyrin C, Baciu M, Segebarth C, Marendaz C (2004) Cerebral regions and hemispheric specialization for processing spatial frequencies during natural scene recognition: an event-related fMRI study. Neuroimage 23:698-707.

Pinel P, Dehaene S (2010) Beyond hemispheric dominance: brain regions underlying the joint lateralization of language and arithmetic to the left hemisphere. J Cogn Neurosci 22:48-66.

Price CJ, Devlin JT (2011) The Interactive Account of ventral occipitotemporal contributions to reading. Trends Cogn Sci 15:246-253.

Price CJ, Mechelli A (2005) Reading and reading disturbance. Curr Opin Neurobiol 15:231-238.

Rossion B, Joyce CA, Cottrell GW, Tarr MJ (2003) Early lateralization and orientation tuning for face, word, and object processing in the visual cortex. Neuroimage 20:1609-1624.
Sabsevitz DS, Medler DA, Seidenberg M, Binder JR (2005) Modulation of the semantic system by word imageability. Neuroimage 27:188-200.

Salmond CH, Ashburner J, Vargha-Khadem F, Gadian DG, Friston KJ (2000) Detecting bilateral abnormalities with voxel-based morphometry. Hum Brain Mapp 11:223-232.

Seghier ML, Price CJ (2010) Reading aloud boosts connectivity through the putamen. Cereb Cortex 20:570-582.

Seghier ML, Lee HL, Schofield T, Ellis CL, Price CJ (2008) Inter-subject variability in the use of two different neuronal networks for reading aloud familiar words. Neuroimage 42:1226-1236.

Seghier ML, Fagan E, Price CJ (2010) Functional subdivisions in the left angular gyrus where the semantic system meets and diverges from the default network. J Neurosci 30:16809-16817.

Seghier ML, Kherif F, Josse G, Price CJ (2011a) Regional and hemispheric determinants of language laterality: implications for preoperative fMRI. Hum Brain Mapp 32:1602-1614.

Seghier ML, Josse G, Leff AP, Price CJ (2011b) Lateralization is predicted by reduced coupling from the left to right prefrontal cortex during semantic decisions on written words. Cereb Cortex 21:1519-1531.

Sharp DJ, Awad M, Warren JE, Wise RJ, Vigliocco G, Scott SK (2010) The neural response to changing semantic and perceptual complexity during language processing. Hum Brain Mapp 31:365-377.

Szwed M, Dehaene S, Kleinschmidt A, Eger E, Valabrègue R, Amadon A, Cohen L (2011) Specialization for written words over objects in the visual cortex. Neuroimage 56:330-344.

Tanaka JW, Curran T (2001) A neural basis for expert object recognition. Psychol Sci 12:43-47.

Tarkiainen A, Helenius P, Hansen PC, Cornelissen PL, Salmelin R (1999) Dynamics of letter string perception in the human occipitotemporal cortex. Brain 122:2119-2132.

Taylor MJ, Arsalidou M, Bayless SJ, Morris D, Evans JW, Barbeau EJ (2009) Neural correlates of personally familiar faces: parents, partner and own faces. Hum Brain Mapp 30:2008-2020.

Twomey T, Kawabata Duncan KJ, Price CJ, Devlin JT (2011) Top-down modulation of ventral occipito-temporal responses during visual word recognition. Neuroimage 55:1242-1251.

Uchida I, Kikyo H, Nakajima K, Konishi S, Sekihara K, Miyashita Y (1999) Activation of lateral extrastriate areas during orthographic processing of Japanese characters studied with fMRI. Neuroimage 9:208-215.

Vigneau M, Jobard G, Mazoyer B, Tzourio-Mazoyer N (2005) Word and non-word reading: what role for the Visual Word Form Area? Neuroimage 27:694-705.

Vinckier F, Dehaene S, Jobert A, Dubus JP, Sigman M, Cohen L (2007) Hierarchical coding of letter strings in the ventral stream: dissecting the inner organization of the visual word-form system. Neuron 55:143-156.

Wang X, Yang J, Shu H, Zevin JD (2011) Left fusiform BOLD responses are inversely related to word-likeness in a one-back task. Neuroimage 55:1346-1356.

Whitaker HA, Ojemann GA (1977) Lateralization of higher cortical functions: a critique. Ann N Y Acad Sci 299:459-473.

Wong AC, Jobard G, James KH, James TW, Gauthier I (2009) Expertise with characters in alphabetic and nonalphabetic writing systems engage overlapping occipito-temporal areas. Cogn Neuropsychol 26:111-127.

Woodhead ZV, Wise RJ, Sereno M, Leech R (2011) Dissociation of sensitivity to spatial frequency in word and face preferential areas of the fusiform gyrus. Cereb Cortex 21:2307-2312.

Xue G, Poldrack RA (2007) The neural substrates of visual perceptual learning of words: implications for the visual word form area hypothesis. J Cogn Neurosci 19:1643-1655.

Xue G, Dong Q, Chen K, Jin Z, Chen C, Zeng Y, Reiman EM (2005) Cerebral asymmetry in children when reading Chinese characters. Brain Res Cogn Brain Res 24:206-214.

Xue G, Chen C, Jin Z, Dong Q (2006) Cerebral asymmetry in the fusiform areas predicted the efficiency of learning a new writing system. J Cogn Neurosci 18:923-931. 